\title{
Behavior genetic analysis of mouse emotionality: II. Stability of factors across genotypes*
}

\author{
WAYNE POLEY and J. R. ROYCE \\ University of Alberta, Edmonton, Alberta, Canada
}

\begin{abstract}
Three emotionally divergent strains of mice (SWR/J, A/HeJ, and SJL/J) were mated to produce three populations: a pure strain population $(N=90)$, an $F_{1}$ population $(N=120)$, and an $F_{2}$ population $(N=96)$. All $S s$ were tested on a battery of measures of emotionality. Each population was factored separately by principal components factoring with varimax and promax rotations. Factorial invariance was assessed quantitatively by congruence coefficients. Eight of the resultant factors were found to be replicable across populations. These were autonomic balance, motor discharge, territorial marking, acrophobia, tunneling-1, tunneling-2, underwater swimming, and audiogenic reactivity. Of the eight factors, motor discharge was most distinct in each population and tunneling-1 was least distinct.
\end{abstract}

The proliferation of behavioral tests in the comparative domain has, as in the field of human differential psychology, produced a need for the development of trait concepts. Traits reduce the number of dimensions in a domain, while the number of tests that could be created is virtually unlimited. The trait approach also has the potential of unveiling the multiple components of individual behaviors.

Previous factor analytic studies with this underlying rationale have been conducted in the domain of rodent emotionality (Willingham, 1956; Furchtgott \& Cureton, 1964; McClearn \& Meredith, 1964; Whimbey \& Denenberg, 1967a, b; Wilcock \& Broadhurst, 1967; Royce, Carran, \& Howarth, 1970; Poley \& Royce, 1970). Research has reached the stage where two criteria have become essential for further progress in the use of factors in comparative psychology: (1) the demonstration that factors are psychologically meaningful, and (2) the demonstration that they are stable or invariant. The first criterion has been met by Royce, Poley, and Yeudall. ${ }^{1}$ The second criterion, factorial invariance, has been dealt with only by Royce (1966a). In Royce's review of the comparative psychological domain, the factors of "autonomic balance" and "motor discharge" were shown to be invariant across species.

The present study was designed to demonstrate further factorial invariance in the area of mouse emotionality, using quantitative as well as qualitative assessments. As such, it represents the second study in a three-part series. The first study dealt with the extraction and identification of factors of mouse emotionality (Royce, Poley, \& Yeudall $^{1}$ ). The third study will deal with the inheritance of factors and estimates of genetic parameters (Royce, Poley, \& Yeudall $^{2}$ ).

*This research represents an extension of a thesis submitted by the first author in partial fulfillment of the $\mathrm{PhD}$ degree at the University of Alberta. It was supported, in part, by N.R.C. Grant APA-105 to the second author.

\section{METHOD}

\section{Subjects}

The Ss were mice from three emotionally contrasted strains and crosses of these strains to produce $F_{1}$ and $F_{2}$ populations. The strains were SWR/J, A/HeJ, and SJL/J (15 males and 15 females in each strain were tested). Breeding stock was obtained from highly inbred Jackson Laboratory lines, and animals tested were derived from matings made at the University of Alberta. The $F_{2}$ generation consisted of the three possible crosses of these strains and their reciprocals (10 males and 10 females in each group). The $F_{2}$ generation consisted of animals from mating the following $F$, crosses with other mice of the same genotype: SWR/J male $\times \mathrm{SJL} / \mathrm{J}$ female, SWR/J male $\times A / \mathrm{HeJ}$ fernale. SJL/J male $\times$ SWR/J female, and $A / \mathrm{HeJ}^{\prime}$ male $\times \mathrm{SJL} / \mathrm{J}$ female. Twelve males and 12 females were tested in each of these groups. Thus, a total of $306 \mathrm{Ss}$ were tested: 90 pure strain Ss, $120 \mathrm{~F}_{1} \mathrm{~s}$, and $96 \mathrm{~F}_{2} \mathrm{~s}$.

The $S$ s were raised under a standard laboratory procedure. They were weaned at 21 days and remained with littermates until 33 days (maximum of five animals in a group). At 33 days, Ss were placed in individual cages, and testing took place from $40 \pm 3$ to $67 \pm 3$ days of age. Animals were housed throughout their life-spans with a 12-h nocturnal-diurnal light cycle, lights being switched on at 9:30 a.m. and off at 9:30 p.m.

\section{Apparatus and Procedure}

At $40 \pm 3$ days of age, Ss began the test sequence with avoidance conditioning, completing at $67 \pm 3$ days of age with underwater swimming. Apparatus and measures have been described more fully elsewhere (Royce, Poley, \& Yeudall ${ }^{1}$ ). The following is a summary of tests and measures in the sequence in which they occur.

\section{Avoidance Conditioning}

This is a two-way shuttlebox with footshock for UCS and electronic buzzer as CS. Parameters used were those determined by Royce (1966b) as optimal for producing strain differences. Footshock is set at $400 \mathrm{~V}$ with CS duration of $3 \mathrm{sec}$ and CS/UCS interval at $3.5 \mathrm{sec}$. Intertrial interval is $120 \mathrm{sec}$. Ss are given 25 consecutive trials per day for 3 days. Two measures are obtained: total correct avoidances and intertrial activity.

\section{Individual Emergence}

In the individual emergence test, the lid of the home cage is 
removed in an observation box and one measure is taken: latency to emerge into the new environment.

\section{Removal Rating}

In this test, the $E$ attempts to remove the animal from its home cage. $S$ is rated on a scale from 0 (no attempt to escape) to 3 (extremely manic).

\section{Activity Wheel}

These are standard activity wheels, proportioned to the size of the species under study. Ss are housed for 2 days in a small living cage adjacent to the wheel, with free access to it. Two consecutive nocturnal readings are averaged, as are two consecutive diurnal readings.

\section{Open Field}

This is one of the most widely used measures of rodent emotionality. It consists of a white circular field, $4 \mathrm{ft}$ in diam, divided into concentric circles, with intersecting lines radiating outward from the center of the field. Five measures are taken, based on averages from 2 consecutive days of testing: latency to leave the start section, activity during a 2 -min trial, penetration into the center of the field, defecation, and urination.

\section{Straightaway}

This is an elevated runway, 50 in. long and 1.5 in. wide. Ss are given a 3-min trial each day for 2 consecutive days. Measures recorded are: latency to leave the start section, activity, defecation, and urination.

Pole

In this test, $S$ is placed on a wire mesh platform, on top of a metal pole 34 in. high. The animal is able to descend of his own volition by a vertical wire mesh ladder. Measures, averaged over a 2-day period, include latency to leave the top, latency to descend, defecation, and urination.

\section{Cell and Hole-in-Wall}

In the cell test, $S$ is placed in a small compartment $(4 \times 4 \times$ 3 in.) with opaque lid and is able to emerge through a small door into a light compartment of the same size. The hole-in-wall test involves emergence from light compartment to dark compartment. Cell is tested on 1 day to yield a.m. and p.m. measures: latency, defecation, and urination. Hole-in-wall is tested the next day (also a.m. and p.m.) to yield the same three measures.

Pipe

The pipe measure consists of two illuminated white plastic boxes connected by an opaque pipe 2 in. in diam. Ss are tested under $14-\mathrm{h}$ food deprivation and obtain two $25-\mathrm{mg}$ food pellets upon entering the goalbox. At each test session, $S$ runs from one goalbox to the other and back again, to yield two trials. Testing is done on 2 consecutive days. This gives six measures: latency to enter pipe, Trial 1; latency to emerge from pipe, Trial 1 ; latency to enter pipe, Trials 2, 3, and 4, averaged; latency to emerge from pipe, Trials 2, 3, and 4, averaged; defecation; and urination. The separation of Trial 1 from Trials 2. 3. and 4 is based on the fact that the animal is unaware of the reward on the first trial; a learning component would be expected to influence later trials.

\section{Circular Activity with Bell}

This apparatus is a circular activity cage. $18 \mathrm{in.}$ in diam
Beneath the cage is a large bell. $S$ is tested on 1 day, during which a $5-\mathrm{min}$ period of silence is followed by a $5-\mathrm{min}$ period of bell ringing; then this sequence is repeated. This gives four measures: activity with bell turned off, activity during bell, defecation, and urination.

\section{Underwater Swimming}

This is a swim tank, $2 \mathrm{ft}$ long $x 3$ in. wide $\times 6$ in. deep. $S$ is immersed in a startbox and swims underwater (a plastic strip prevents surfacing), to emerge on the other side. Five trials for 3 consecutive days yield two measures: latency to enter (leave starting cage) and latency to traverse the swimway.

\section{Analysis}

Of the 37 measures described above, only 27 were retained for the present analysis. They were retained because they assessed the eight most interpretable factors of emotionality (Royce, Poley, \& Yeudall ${ }^{1}$ ). The 27 measures were intercorrelated separately for the three populations under study.

Principal components factoring was used with ones inserted in the principal diagonal. ${ }^{3}$ Factors were rotated to orthogonal simple structure by varimax, followed by rotation to oblique simple structure by promax $(K=2)$. Factors were compared across mating plans by congruence coefficients. The formula for the congruence coefficients is $r_{c}=\Sigma a b / \sqrt{\Sigma a^{2} \Sigma b^{2}}$, where $a$ and $b$ are corresponding factor loadings of the two populations. Thus, $r_{c}$ is not dissimilar from the Pearson product-moment correlation.

\section{RESULTS}

Factoring was terminated with eigenvalues greater than 1.0. This yielded 9 factors for each of the pure strain and $F_{1}$ populations and 10 factors for the $F_{2}$ population (Table 1). Based on the loadings from Royce, Poley, and Yeudall ${ }^{1}$, we could expect to find 8 interpretable factors in the present analysis. The additional 1 or 2 factors can be explained either by overfactoring or by the retention of common factor variance from the less interpretable factors. The 8 factors expected in the present analysis include motor discharge, autonomic balance, territorial marking, tunneling-1, tunneling-2, acrophobia, audiogenic reactivity, and underwater swimming. Motor discharge is defined primarily as a freezing in open light factor with major loadings from open field and straightaway measures of locomotion. Autonomic balance is identified primarily by defecation loadings from several tests, while territorial marking is identified primarily by urination loadings from several tests. Tunneling-1 is defined by defecation loadings from tests involving passage through a small doorway or tunnel, while tunneling- 2 is derived from the same tests but involves primarily measures of locomotion. Although the last 3 factors are apparatus-dependent. they are not necessarily artifactors. These include acrophobia from the pole test. audiogenic reactivity from the bell test, and underwater swimming from the test with the same name.

The factors recovered (Table 1) can be compared across mating plans by qualitative means: that is, by trying to identify the same factor from its major 


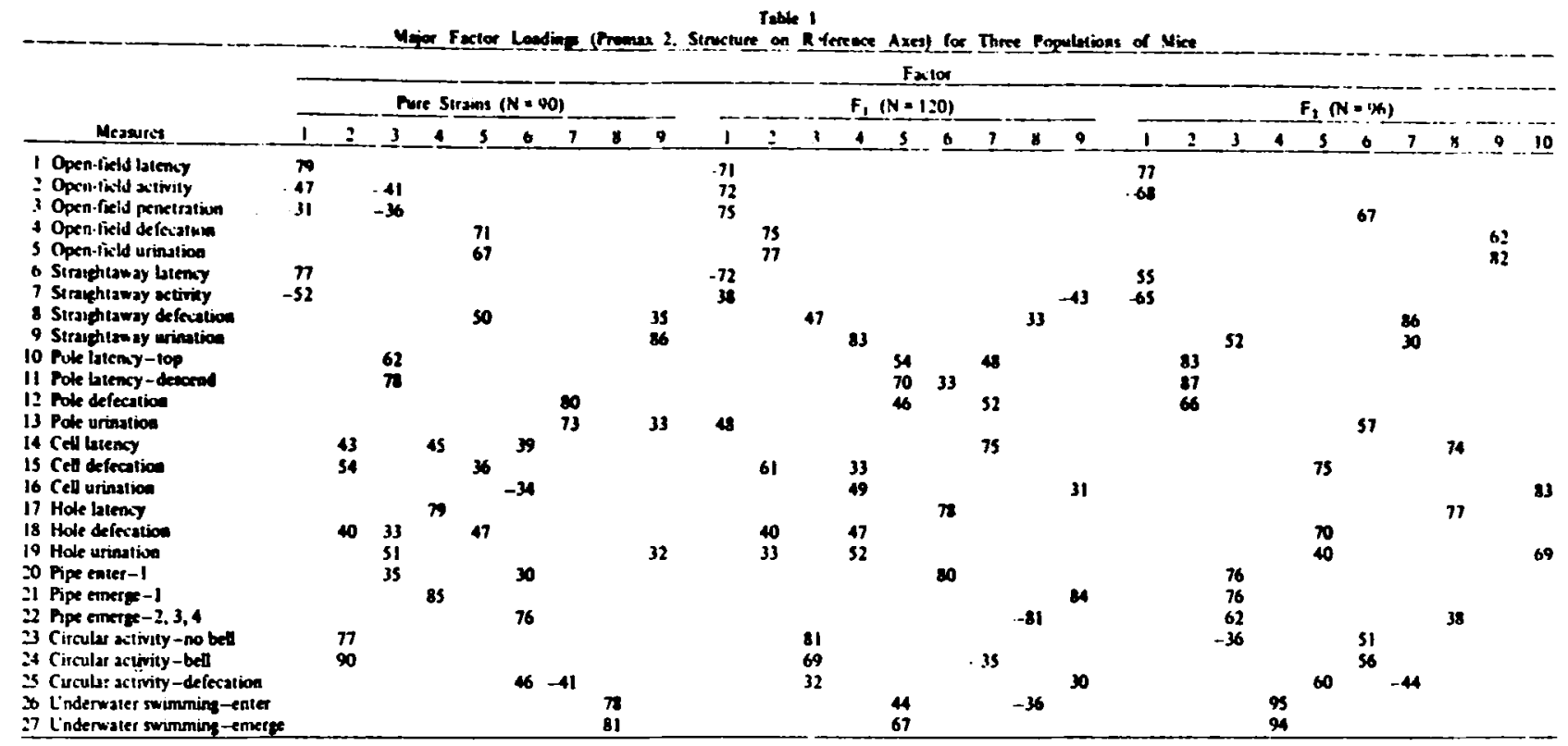

loadings. This has been supplemented by invariance coefficients (Table 2), which yield similar matchings.

The motor discharge factor gives the best matching across all three mating plans. In each case, it appears as the first factor with congruence coefficients (Table 2), ranging from .71 to .82 . Autonomic balance can be identified as Factor 5 of pure strains, best matching Factor 2 for $F_{1}$ Ss and Factor 9 of the $F_{2}$ population. Congruence coefficients range from .54 to .84 , across three mating plans, for this factor. Some urination measures also load on the factor, as might be expected from the high correlation between autonomic balance and territoriality found by Royce, Poley, and Yeudall. ${ }^{1}$ Territorial marking itself is best identified as Factor 9 of pure strains, Factor 4 of $F_{1}$, and Factor 10 of $F_{2}$. Congruences range from .32 to .65 .

Tunneling- 1 and tunneling- 2 can also be traced across the three mating plans. Tunneling-1 (defined primarily by elimination measures) appears as Factor 2 of pure strains (fusing with audiogenic reactivity), Factor 4 of $F_{1}$ (fusing with territorial marking), and Factor 5 of $F_{2}$. Congruences range from .34 to .37 . However, the presence of higher matchings with other factors indicates some difficulty in clearly separating this factor from others. Tunneling-2 (defined primarily by activity) appears as Factor 4 of pure strains, Factor 6 of $F_{1}$, and Factor 8 of $F_{2}$. Congruences range from .38 to .58 .

The three experimentally dependent factors also can be matched across mating plans. Acrophobia is identified as Factor 3 of pure strains, Factor 5 for $F_{1}$, and Factor 2 for $F_{2}$. Congruences range from .54 to 64 . Audiogenic reactivity fuses with tunneling-1 for pure strains (Factor 2) but is more distinct for $F_{1}$ as Factor 3 and $F_{2}$ as Factor 6 . Congruences range from .43 to .69 . Similarly, underwater swimming appears clearly as Factor 8 for pure strains but fuses with acrophobia (Factor 5) in $F_{1}$ Ss and is again distinct as Factor 4 in
$F_{2}$. Congruences range from .48 to .91 .

\section{DISCUSSION}

The major purpose of this research was to demonstrate the stability of factors under a specified range of conditions: different mating plans. Variation in the general genetic characteristics of these populations might be expected to seriously disrupt the organization of behavior. For example, the inbred strains of the pure strain population are expected to be $99 \%$ homozygous (Falconer, 1960) following the more than 20 generations of brother-sister matings which have produced these strains. Inbreeding also produces loss of vigor in a variety of characteristics, i.e., inbreeding depression. Whereas pure strains are homozygous, $F_{1}$ animals will be heterozygous at many loci. Frequently, $F_{1}$ populations will manifest "hybrid vigor" or a recovery of the functions depressed by inbreeding (Bruell, 1967). Differences in the variance of behaviors are often observed between pure strain and $F_{1}$ populations. While physical characteristics typically show reduced variance in the $F_{1}$ population, McClearn (1967) has suggested that behavioral characteristics may show increased variance. The $F_{2}$ population might be expected to have an even more disruptive influence on organization. It is here that the segregation of genes may be influenced by linkage, with a direct influence on correlation coefficients (Thompson, 1957).

All of the above conditions lead one to suspect that qualitative changes in the organization of behavior may be a consequence of different mating plans. If this were so, quantitative geretic analyses of factor scores would be difficult. That is, there would be no continuity of the same factor across mating plans for purposes of analysis. This study has demonstrated that factors do, in fact, appear in recognizable form across these conditions. On 
Table 2

Congruence Coefficients Between Populations for Factors of Emotionality*

\begin{tabular}{|c|c|c|c|c|c|c|c|c|c|}
\hline \multirow{2}{*}{$\begin{array}{c}\text { Pure } \\
\text { Strain } \\
\text { Factors }\end{array}$} & \multicolumn{9}{|c|}{$F_{1}$ Factors } \\
\hline & 1 & 3 & 5 & 6 & 2 & 7 & 8 & 9 & 4 \\
\hline 1 & -81 & -12 & -08 & 13 & 05 & 10 & 02 & 11 & 05 \\
\hline 2 & 00 & & 01 & 01 & 16 & 01 & -21 & 00 & 37 \\
\hline 3 & -33 & -10 & 54 & 36 & 10 & 23 & -07 & 17 & 28 \\
\hline 4 & -01 & -05 & 06 & 56 & -07 & 26 & -06 & 48 & 00 \\
\hline 5 & -03 & 07 & -12 & -10 & 84 & 00 & 15 & 03 & 32 \\
\hline 6 & -15 & 15 & 02 & 09 & 01 & 46 & -36 & 09 & -13 \\
\hline 7 & 21 & 05 & 14 & 22 & 08 & 35 & 16 & -11 & 14 \\
\hline 8 & 01 & 11 & 48 & -13 & -09 & 00 & -17 & 10 & 21 \\
\hline 9 & 12 & 14 & -03 & -05 & 06 & 10 & 40 & 02 & 65 \\
\hline
\end{tabular}

\begin{tabular}{|c|c|c|c|c|c|c|c|c|c|c|}
\hline \multirow{2}{*}{$\begin{array}{c}\text { Pure } \\
\text { Strain } \\
\text { Factors }\end{array}$} & \multicolumn{10}{|c|}{$\mathrm{F}_{2}$ Factors } \\
\hline & 1 & 6 & 2 & 8 & 9 & 5 & 7 & 4 & 10 & 3 \\
\hline 1 & 82 & -08 & 15 & 06 & 04 & 01 & -03 & -06 & 06 & 11 \\
\hline 2 & -01 & 43 & -09 & 19 & -32 & 37 & -21 & 04 & 24 & -36 \\
\hline 3 & 26 & -25 & 59 & 03 & -06 & 30 & 05 & -04 & 29 & 31 \\
\hline 4 & 13 & -08 & 15 & 58 & -04 & -06 & -14 & 04 & -06 & 48 \\
\hline 5 & 01 & -10 & 03 & -08 & & 40 & 21 & 01 & 25 & -07 \\
\hline 6 & 14 & -09 & 05 & 36 & -19 & 38 & -01 & -01 & -32 & 33 \\
\hline 7 & 24 & 19 & 29 & 17 & 03 & -03 & 25 & -03 & 09 & 02 \\
\hline 8 & 00 & 07 & -05 & 01 & 02 & 13 & -04 & & 17 & 02 \\
\hline 9 & 14 & 32 & -03 & -05 & 04 & 09 & 35 & -02 & 32 & 21 \\
\hline \multirow{2}{*}{$\underset{\text { Factors }}{F_{1}}$} & \multicolumn{10}{|c|}{$\mathrm{F}_{2}$ Factors } \\
\hline & 1 & 9 & 6 & 10 & 2 & 3 & 8 & 4 & 7 & 5 \\
\hline 1 & -71 & 06 & 35 & -14 & -14 & 07 & -01 & 00 & 00 & -06 \\
\hline 2 & 04 & 59 & 04 & 24 & 17 & -07 & -06 & -03 & -05 & 48 \\
\hline 3 & -14 & -36 & 49 & 11 & 13 & -30 & -01 & 15 & -03 & 19 \\
\hline 4 & 16 & 00 & 04 & 62 & -23 & 11 & 03 & 20 & 25 & 34 \\
\hline 5 & 06 & -06 & -02 & -01 & 64 & 13 & -14 & 53 & 00 & 18 \\
\hline 6 & 13 & -18 & -05 & -03 & 27 & 45 & 38 & -12 & -06 & 00 \\
\hline 7 & 23 & 17 & -08 & -12 & 31 & 16 & 60 & -01 & 02 & 13 \\
\hline 8 & 20 & 07 & 05 & 01 & 08 & -22 & -37 & -21 & 25 & -01 \\
\hline 9 & 11 & -01 & -07 & 19 & 12 & 33 & 12 & -02 & -35 & 24 \\
\hline
\end{tabular}

*Factors are rearranged by columns to produce the best matching (maximize coefficients in the principal diagonal).

the other hand, it is also clear that the present study has not refuted the claims of Thompson (1957), Hirsch (1967), and McClearn (1967) that individual correlations may be influenced by mating plans. Furthermore, a study explicitly designed for these purposes would be required in order to assess these claims.

The present study has demonstrated the invariance of eight factors of emotionality, both qualitatively and quantitatively. Qualitative assessments have been based on the ability to recognize (interpret) the same factor in different mating plans. They have been further supported by quantitative indices of invariance. The fusion and splitting of some of the factors does not detract from the present demonstration of invariance.
Fusion refers to the combination of closely related factors into a single broad trait. Splitting is the opposite of this-the breakdown of a factor into narrower elements. These processes are the expected consequences of having correlated factors (Royce, Poley, \& Yeudall ${ }^{1}$ ). When the correlations between factors are strong, as will be the case in some populations, it is difficult to separate factors and fusion will result. Evidence for this difficulty is readily apparent in Table 1 . In addition, the presence of high off-diagonal congruence coefficients in Table 2 is indicative of both factor fusion and factor splitting. (This phenomenon, however, does not indicate an absence of invariance. which would be manifested in a table of zero congruences.) In these cases. a broad factor 
will be correlated with the components which split off from it, or fuse to form the more inclusive trait. Thus, we cannot expect the 1:1 relationship which would be manifested in Table 2 by perfect matching for diagonal elements and zero matching for off-diagonals. The differentiation involved here is more than a statistical artifact. It may have further implications for developmental processes or hierarchical trait models. However, further research will be required to assess these possibilities.

Implications for further comparative research with factors are: (1) factors are sufficiently stable to permit quantitative genetic analysis, and (2) the systematic study of factors via bivariate designs as recommended by Royce (1950) should be feasible. It should be pointed out, however, that experimental conditions which are particularly severe (e.g., drugs, stress) may reorganize behavior' as well as quantitatively change factors. In these cases, it may be necessary to study the effects using both bivariate and multivariate designs.

\section{REFERENCES}

Bruell, J. H. Behavioral heterosis. In J. Hirsch (Ed.), Behavior-genetic analysis. New York: McGraw-Hill, 1967.

Falconer, D. S. Introduction to quantitative genetics. Edinburgh and London: Oliver and Boyd, 1960.

Furchtgott, E., \& Cureton, E. E. Factor analysis of emotionality and conditioning in mice. Psychological Reports, 1964, 15, 787-794.

Hirsch, J. Behavior-genetic or "experimental" analysis: The challenge of science versus the lure of technology. American Psychologist, 1967, 22, 118-130.

MoClearn, G. E. Genes, generality and behavior research. In J. Hirsch (Ed.), Behavior-genetic analysis. New York: McGraw-Hill, 1967.

McClearn, G. E., \& Meredith, W. Dimensional analysis of activity and elimination in a genetically heterogeneous group of mice. A nimal Behaviour, 1964, 12, 1-10.

Poley, W., \& Royce, J. R. Genotype, maternal stimulation and factors of mouse emotionality. Journal of Comparative \& Physiological Psychology, 1970, 71, 246-250.
Royce. J. R. A synthesis of experimental designs in program research. Journal of General Psychology, 1950, 43, 295-303.

Royce, J. R. Concepts generated in comparative and physiological psychological observations. In R. B. Cattell (Ed.), Handbook of multivariate experimental psichology. Chicago: Rand McNally, 1966a.

Royce, J. R. Optimal stimulus parameters in avoidance conditioning of inbred strains of mice. Multivariate Behavioral Research, 1966b, 1, 209-217.

Royce, J. R., Carran, A. B., \& Howarth, E. Factor analysis of emotionality in ten inbred strains of mice. Multivariate Behavioral Research, 1970, 5, 19-48.

Thompson, W. R. Traits, factors and genes. Eugenics Quarterly, 1957, 4, 8-16.

Whimbey, A. E., \& Denenberg, V. H. Two independent behavioral dimensions in open-field performance. Joumal of Comparative \& Physiological Psychology, 1967a, 63, 500-504.

Whimbey, A. E., \& Denenberg, V. H. Experimental programming of life histories: The factor structure underlying experimentally created individual differences. Behaviour, $1967 \mathrm{~b}, 29,296-314$.

Wilcock, J., \& Broadhurst, P. L. Strain differences in emotionality: Open-field and conditioned avoidance behavior in the rat. Journal of Comparative \& Physiological Psychology, 1967, 63, 335-338.

Willingham, W. W. The organization of emotional behavior in mice. Journal of Comparative \& Physiological Psychology, $1956,49,345-348$.

\section{NOTES}

1. Royce, J. R., Poley, W., \& Yeudall, L. T. Behaviour genetic analysis of mouse emotionality, I: The factor analysis. Submitted for publication.

2. Royce, J. R., Poley, W., \& Yeudall, L. T. Behaviour genetic analysis of mouse emotionality, III: The genetic analysis. Unpublished manuscript, 1972.

3. Different factoring procedures have been employed in our research with mouse emotionality. This provides further evidence for the robustness of the factors obtained. For example, Royce, Poley, \& Yeudall $^{1}$ used alpha factoring with squared multiple correlations as initial communality estimates and iterating to convergence.

(Received for publication September 1, 1972; accepted November 10,1972.) 